\title{
Consensus statement on the management of the GH-treated adolescent in the transition to adult care
}

\author{
P E Clayton, R C Cuneo ${ }^{1}$, A Juul ${ }^{2}$, J P Monson ${ }^{3}$, S M Shalet ${ }^{4}$ and M Tauber ${ }^{5}$ \\ Endocrine Science Research Group, Stopford Building, University of Manchester, Oxford Road, Manchester M13 9PT, UK, ${ }^{1}$ Metabolic Research Unit, \\ University of Queensland and the Department of Diabetes and Endocrinology, Princess Alexandra Hospital, Brisbane, Australia, ${ }^{2}$ Department of Growth \\ and Reproduction, Rigshospitalet, Copenhagen, Denmark, ${ }^{3}$ St Bartholomew's Hospital, London, UK, ${ }^{4}$ Christie Hospital, Manchester, UK and ${ }^{5}$ Hôpital des \\ Enfants, Toulouse, France
}

(Correspondence should be addressed to P E Clayton; Email: peterclayton@man.ac.uk)

\begin{abstract}
The European Society for Paediatric Endocrinology held a consensus workshop in Manchester, UK in December 2003 to discuss issues relating to the care of GH-treated patients in the transition from paediatric to adult life. Clinicians experienced in the care of paediatric and adult patients on GH treatment, from a wide range of countries, as well as medical representatives from the pharmaceutical manufacturers of $\mathrm{GH}$ participated.
\end{abstract}

European Journal of Endocrinology 152 165-170

\section{Introduction}

Growth hormone $(\mathrm{GH})$ has multiple beneficial effects in addition to its promotion of linear growth. These include maintenance of normal body composition and metabolism through adult life. Therefore, in GHdeficient individuals replacement throughout the life cycle is indicated. However, up until recently, insufficient evidence was available to determine if $\mathrm{GH}$ treatment should continue seamlessly or could be interrupted for a period of time at the end of growth without incurring disadvantage to the adolescent. Equally, information on GH dose requirement has only now become available. Thus the European Society for Paediatric Endocrinology judged that this was an appropriate time to provide some guidance on the assessment of those completing treatment with $\mathrm{GH}$ for growth promotion, not only for GH deficiency (GHD) but also for other causes of short stature.

Clinical programmes need to be developed for these young people to facilitate the orderly transfer of their care from paediatric to adult endocrine services, to optimise compliance and patient acceptance and to minimise interruption of care. This transfer of care occurs during a developmental stage referred to as the transitional period, during which important issues relating to post-pubertal maturation require consideration.

\section{Definition of transition}

Transition refers to a broad set of physical and psychosocial changes, arbitrarily defined as starting in late puberty and ending with full adult maturation. This usually implies a period from mid to late teens until 6-7 years after achievement of final height.

The aims of management in the transition period for the GH-treated adolescent include the following: (a) reassessment of aetiology and disease-specific management; (b) reassessment of the $\mathrm{GH}$ treatment regimen to mimic the diminishing production of endogenous GH secretion; (c) achievement of full adult somatic development including lean body mass and bone mineral accrual; (d) completion of pubertal, sexual and reproductive maturation; (e) reduction of metabolic and cardiovascular risks; (f) attainment of adult psychosocial development; and (g) education to ensure that patients have an understanding of their disease to develop autonomy in health care decision making.

\section{Delivery of transition care}

Clinics in the transition period have been seen to be effective in a number of chronic disease states, such as diabetes mellitus, chronic renal failure and cystic fibrosis, in terms of improving compliance during the hand-over to adult services, and aiding patient acceptance of adult services. Transition care requires a dedicated service with contributions from paediatric and adult endocrinology. Local resources will determine the precise format of the service.

Paediatric and adult endocrinologists participating in a transition programme should be experienced in the management of hypopituitarism and GHD. The multidisciplinary approach should include the support of 
specialised nurses, reproductive specialists, psychologists and social workers. This shared responsibility will lead to improved patient care. Broadening of training opportunities and development of joint research projects are additional benefits.

Patient education is an implicit part of the transition programme. This educational process should have commenced at the time of diagnosis in childhood, when the patient and family are informed not only of the linear growth-stimulating effect of $\mathrm{GH}$ but also its life-long effects on body composition and metabolism.

\section{Tasks for a transition care programme}

\section{Reassessment of pituitary status}

$\mathrm{GH}$ secretion and insulin-like growth factor-I (IGF-I) levels reach a maximum at mid to late puberty and subsequently decline. This decline is rapid until the mid 20s. The GH replacement strategy differs in childhood from that adopted in adult life: in childhood all degrees of GHD are considered for replacement whereas in adult life only patients with severe GHD are currently treated. Therefore there is a requirement for re-evaluation of the diagnosis when the major paediatric goals of treatment have been reached. Thus, in adolescents with GHD, treatment should be stopped for reevaluation of the diagnosis when growth and pubertal development are considered to be complete.

All patients require re-evaluation of pituitary function during transition (with the only exception being those with severe congenital or acquired panhypopituitarism). The interval between the re-evaluation and the discontinuation of $\mathrm{GH}$ should not be less than one month. GH reserve is assessed by measurement of a serum IGF-I concentration and/or a GH stimulation test. The tests currently recommended are the insulin tolerance test (ITT) with the arginine or glucagon tests as alternatives (1). The GH-releasing hormone (GHRH)-arginine test may be unreliable in patients with suspected hypothalamic disease. The GHRH and clonidine tests alone are not useful.

The extent of GH-IGF-I re-evaluation depends on the a priori likelihood of profound GHD (Fig. 1). Thus we propose the definition of two groups of patients: (a) high likelihood - those with severe GHD in childhood with or without two or three additional hormone deficits, which may be due to a defined genetic cause, those with severe GHD due to structural hypothalamic-pituitary abnormalities, central nervous system (CNS) tumours or patients having received high-dose cranial irradiation and (b) low likelihood - the remaining patients, including those with idiopathic GHD, either isolated or with one additional hormone deficit.

In patients with a high likelihood of persistent GHD, an IGF-I value $\leq-2$ s.D. score (SDS) off GH treatment for at least 4 weeks should be considered sufficient evidence of profound GHD. If the IGF-I is $>-2$ SDS, a GH

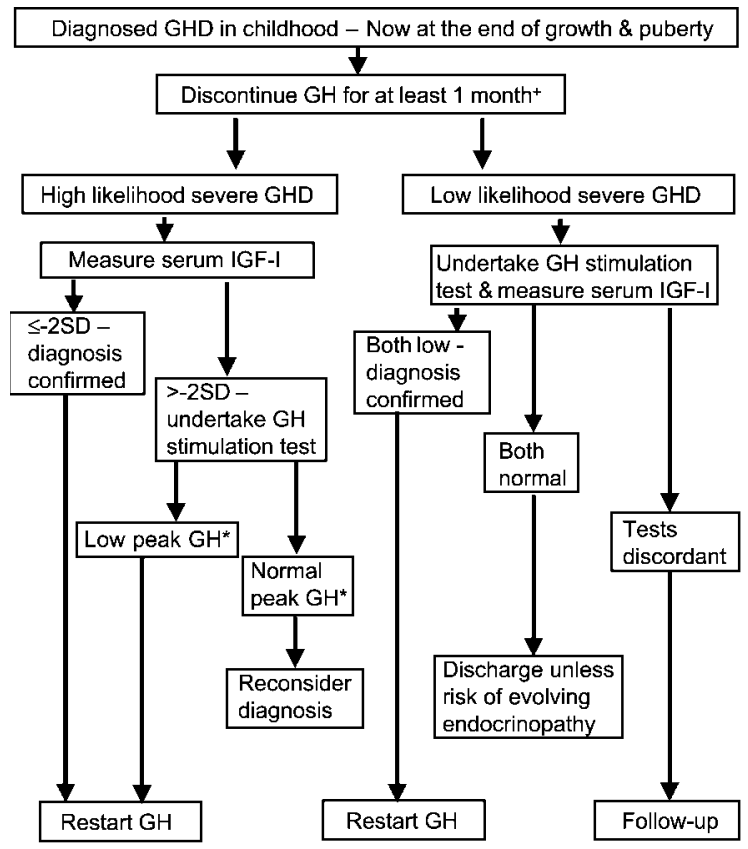

Figure 1 The process of re-evaluation of GH and IGF-I levels at the end of growth in those with GHD diagnosed in childhood (see text for further details). ${ }^{*}$ Peak $\mathrm{GH}<5 \mu \mathrm{g} / \mathrm{l}$; + For those with severe congenital or acquired panhypopituitarism (4 or 5 hormone deficiencies), $\mathrm{GH}$ can be continued without interruption.

provocation test should be performed. If this shows a low GH response, the diagnosis of GHD is reconfirmed. If the peak $\mathrm{GH}$ is above the appropriate cut-off value, the diagnosis should be reconsidered.

Patients with a lower likelihood of retesting GH deficient should have an IGF-I measurement and one $\mathrm{GH}$ provocation test. If both are low, the diagnosis of GHD is reconfirmed. If both are normal, the patient can be discharged unless they are at risk of evolving endocrinopathy, such as those who received prior CNS irradiation. If the tests are discordant, the patient should be followed-up.

Severe GHD in adults has been defined as a peak $\mathrm{GH}$ response $<3 \mu \mathrm{g} / \mathrm{l}$ in response to an ITT and is an accepted criterion for $\mathrm{GH}$ replacement therapy in adults (1). However, this definition is likely to be too conservative in the transition period. In normal children, the most exuberant GH response to a stimulation test occurs in late puberty with GH levels inevitably exceeding $5 \mu \mathrm{g} / \mathrm{l}$. Thus we propose the criteria for severe GHD in the transition period to be $<5 \mu \mathrm{g} / \mathrm{l}$ in response to a GH stimulation test. Standardisation and quality control of GH assays are critical $(1,2)$.

On re-evaluation of the population of adolescents who received GH replacement for childhood GHD, a proportion will not be severely $\mathrm{GH}$ deficient but will fail to attain normal $\mathrm{GH}$ status (peak GH level $<10 \mu \mathrm{g} / \mathrm{l}$ but $\geq 5 \mu \mathrm{g} / \mathrm{l})(2,3)$. It is apparent that a significant number of these patients will be reluctant 
to comply with long-term follow-up. However, the possibility of an evolving endocrinopathy justifies continued surveillance.

Before testing, adequate hormone replacement is required. Full pituitary function testing is not required in patients already diagnosed and substituted for complete combined pituitary deficiency. Oral oestrogen may decrease IGF-I levels. This should be taken into consideration. However, the effect of oestrogen on the efficacy of diagnosing GHD in the transition patient has not been evaluated. In other cases basal testing should be performed regularly, particularly in patients at high risk for evolving hormonal deficits, i.e. genetic defects, hypothalamic pituitary malformations, postCNS tumour surgery, infiltrating tumours, autoimmune diseases and irradiated patients. Oestrogen should be stopped at least 4 weeks prior to evaluation of the adrenal axis due to its effect on cortisol-binding globulin.

Any patient with severe GHD should have cranial and pituitary magnetic resonance imaging (MRI) evaluated according to a standardised protocol, including measurements of pituitary height and search for CNS malformations. Many will have had MRI at diagnosis in childhood, and this does not need repeating.

Genetic advice and testing, if not already offered, should be considered in familial cases, multiple pituitary hormone deficiency or severe isolated GHD of unclear aetiology.

\section{The decision to reinstitute GH treatment}

It is known that normal maturation of muscle mass and achievement of peak bone mass occur during the transition phase and are $\mathrm{GH}$ dependent. There are now also a number of controlled trials of up to 2 years duration in older adolescents and young adults with severe GHD showing the negative consequences of interrupting $\mathrm{GH}$ replacement and the positive effects of continued treatment on fat distribution, muscle mass and function, cardiac structure and performance and bone mass (4-9). In the light of these data it seems appropriate to offer to reinstitute $\mathrm{GH}$ replacement in all adolescents with the diagnosis of severe GHD. In adolescents who decline the offer to continue on GH replacement, monitoring of GH-dependent endpoints (see below) is required. Evidence of subsequent deterioration constitutes the basis for reassessment of the decision to reinstate $\mathrm{GH}$ therapy.

\section{Management of GH replacement}

The transition phase is an appropriate time to modify $\mathrm{GH}$ dosages. In this regard, the $\mathrm{GH}$ replacement dose should be adjusted on the background of the physiological decline of $\mathrm{GH}$ and IGF-I levels, which takes place after late puberty. Additionally, the sexual dimorphism of GH secretion and sensitivity has clinical implications for dosing strategy.
During puberty, patients will have received GH treatment at a wide range of doses, ranging from 25 to $50 \mu \mathrm{g} / \mathrm{kg}$ per day (and in some cases higher) (2). In the transition phase, patients should restart $\mathrm{GH}$ at a dose of $0.2-0.5 \mathrm{mg} /$ day, with subsequent dose adjustments on the basis of IGF-I determinations using age- and gender-specific normative data. A final maintenance dose in excess of $2 \mathrm{mg} /$ day is rarely required. It is recognised that there are patients with GHD who do not normalize IGF-I levels despite a good clinical response, and thus do not require dose escalation. Women generally require higher GH doses than men, in particular those treated with oral oestrogen replacement; the dose and route of oestrogen administration influences IGF-I generation and therefore $\mathrm{GH}$ dose requirement $(10,11)$.

IGF-I measurement is at present the best biochemical marker of $\mathrm{GH}$ responsiveness and is mandatory as a safety marker (12). The GH dose should be adjusted to achieve IGF-I levels within but not exceeding the upper normal range (IGF-I SDS 0 to +2 ). Serum IGFI levels should be monitored every 6 months.

Measurements of IGF-binding protein (IGFBP)-3 levels do not provide substantial additional information regarding responsiveness to $\mathrm{GH}$. However, further data could be helpful to investigate the potential association of the IGF-I/IGFBP-3 ratio with the risk of developing malignancies later in life (12).

\section{Management of underlying disease and optimisation of other hormone replacement}

Clinical parameters for long-term follow-up After paediatric $\mathrm{GH}$ treatment in severe GHD, height achieved approaches target height. In contrast there remains a substantial deficit with respect to lean body mass and bone mineral content (13). Lipoprotein abnormalities have not been highlighted in the transition period but this probably reflects the young age of these patients and the effects of prior GH therapy.

It is also recognised that such adolescents may develop impaired quality of life (QoL) experiencing psychosocial problems related to GHD and/or the underlying chronic disease, for which they may need counselling. Thus follow-up of this aspect is advised. There is, however, a pressing need for validated instruments to assess QoL in this age group.

We recommend the following minimum observations: height, weight, body mass index and waist and hip circumference yearly; blood pressure, heart rate and QoL assessment yearly; bone densitometry and lipids at baseline and at 2- to 5-year intervals.

The attainment of a normal peak bone mass defined as a $\mathrm{T}$ score $>-1$ (i.e. standardised to average adult peak bone mass) is one of the therapeutic objectives during the transition period. Bone density data should be interpreted using age-related $\mathrm{Z}$ scores as well as $\mathrm{T}$ scores during the teenage years and using $\mathrm{T}$ scores 
for those in their 20s. Lower values may be found in those with significant short stature (height SDS $<-2$ ), as $\mathrm{Z}$ and $\mathrm{T}$ scores are height dependent. Assessment using a derived volumetric bone density may be required in these individuals (14).

In view of the known effects of GH on carbohydrate metabolism (12), as an additional safety procedure, we advise measurement of fasting plasma glucose, insulin and glycated haemoglobin (HbA1C). Glucose tolerance testing may be considered in obese patients and those with a family history of diabetes mellitus.

Guidelines for CNS imaging Patients with treated intracranial malignancy require annual imaging with MR for at least 3 years after primary tumour therapy and subsequently as appropriate. Patients with treated craniopharyngioma or pituitary adenoma should be reimaged at a frequency determined by the perceived risk of regrowth of the tumour.

Thyroxine and glucocorticoid replacement The diagnosis of central or primary hypothyroidism should be based on serum free thyroxine (FT4) and thyrotrophin. Because of the risk of subtle central hypothyroidism in these patients, if the FT4 is in the lower third of the reference range, a body of opinion believes that commencement of L-thyroxine, aiming to achieve a serum FT4 in the upper half of the reference range, is appropriate.

The GH-treated adolescent already on hydrocortisone replacement should continue this treatment with a daily dose of $8-12 \mathrm{mg} / \mathrm{m}^{2}$, in a thrice daily regimen (early morning, lunch, evening). This may be monitored and adjusted by means of a modified day curve of serum or salivary cortisol (15). GH replacement may accelerate cortisol metabolism (16), which may increase the risk of cortisol insufficiency in those with untreated subtle degrees of adrenocorticotrophin deficiency or those on suboptimal hydrocortisone replacement.

Oestrogen and androgen replacement Adequate gonadal hormone replacement in conjunction with $\mathrm{GH}$ is critical for optimising somatic development. Oestrogen may be replaced by the transdermal or oral routes dependent on patient preference. Female patients may additionally require androgen supplementation for promotion of pubic hair growth and libido, but more data are required in this age group. As part of the transition management, fertility issues should be discussed in collaboration with a reproductive specialist.

In males, testosterone may be replaced by intramuscular injection, transdermal preparations or implant. Spermatogenesis and testicular growth may be induced by pulsatile gonadotrophin-releasing hormone or with human chorionic gonadotrophin usually combined with follicle-stimulating hormone.

\section{Management of non-GHD conditions}

Non-GHD conditions treated with GH during childhood include Turner syndrome, Prader-Willi syndrome (PWS), small for gestational age (SGA), chronic renal insufficiency (CRI) and idiopathic short stature (ISS). We have considered which conditions require transitional care and what investigations are required. Not all require endocrine transition care, but all children treated with GH should be considered for inclusion into a surveillance programme (see below).

Turner syndrome has numerous life-long health issues such as reproductive dysfunction, cardiovascular disease, osteoporosis and obesity, which require transitional care (17). With regard to their previous GH therapy, these patients require no specific investigations. Endocrine evaluation should include assessment of adult height, thyroid status, hormonereplacement therapy requirements, carbohydrate metabolism and bone mass (18).

PWS has health consequences including, obesity, hypogonadism, hypotonia, developmental disability and sleep apnoea. This patient group requires transitional care. A proportion of these patients have GHD and respond to GH therapy during childhood with regard to growth, lean mass, muscle strength and fat mass. Evidence that GH therapy should continue through the transition period is lacking and further research studies are required. Endocrine evaluation should include assessment of carbohydrate metabolism, gonadal and thyroid status.

SGA and ISS patients who have received GH treatment during childhood should be followed to ascertain adult height, although GH therapy may have been discontinued prior to final height. These patients do not require specific transitional care with regard to prior GH treatment. SGA patients may require surveillance for metabolic problems.

CRI patients will be followed-up by nephrologists, and no specific endocrine transitional care is required.

In the above indications for $\mathrm{GH}$ therapy final adult height achieved is not well documented and for this reason should be measured in all patients during transition care. IGF-I should have been monitored during GH therapy and there is evidence that following the cessation of GH therapy the GH/IGF-I axis will return to its pretreatment status $(19,20)$. For this reason measurement of IGF-I post-treatment is not essential.

\section{Surveillance programmes}

Programmes, independent of direct clinical care, are required to identify the long-term consequences of childhood GH therapy. It is recognised that to exclude an increased incidence of malignancy, diabetes mellitus or other rare adverse events will require large patient cohorts, prolonged periods of follow-up and expected background population disease rates. These 
surveillance programmes will need the support of government agencies, endocrine societies and industry.

\section{Conclusions}

The period from late adolescence through early adulthood is characterised by major physical and psychosocial change. This places a particular strain on a young person with a chronic disorder, such as hypopituitarism, and highlights the need to develop specific multidisciplinary services, which call on the expertise of paediatric and adult endocrinologists and nurse specialists as well as reproductive medicine, psychologists and social workers, to manage such individuals. A plan of care from the late teenage years to the mid 20 s for those adolescents who have been receiving GH treatment through childhood has been proposed. This is a time when the requirement for GH treatment needs to be re-evaluated. For those continuing on $\mathrm{GH}$, a treatment regimen to optimise somatic growth (body composition and bone density), cardiovascular function and QoL needs to be delivered, while for those discontinuing GH, long-term surveillance is recommended.

\section{Acknowledgements}

This statement is the result of a Consensus Workshop organised by the European Society of Paediatric Endocrinology in Manchester, UK in December 2003. The other participants at the workshop were: K Albertsson-Wikland, Pediatric Growth Research Centre, Goteborg (Sweden), A Attanasio, Turin (Italy), K Attie, Rio de Janeiro (Brazil), W Blum (Lilly), R Brauner, Hôpital Necker-Enfants Malades, Paris (France), F Cassorla, University of Chile, Santiago (Chile), J Christiansen, Kommunehospitalet, Århus (Denmark), A M Colao, Federico II University of Naples (Italy), C Cowell, The Children's Hospital at Westmead, Sydney (Australia), S Ezzat, Mount Sinai Hospital, Toronto (Canada), M Foldager (Ferring), Y Hasegawa, Tokyo Metropolitan Kiyose Children's Hospital (Japan), S Ispas-Jouron (Lilly), G Johannsson, Sahlgrenska University Hospital, Goteborg (Sweden), J Jorgensen, Århus Kommunehospital, Århus (Denmark), A-M Kappelgard (Novo Nordisk), S de Muinck Keizer, Sophia Kinderziekenhuis, Rotterdam (The Netherlands), A Ferrandez Longas, Hospital Infantil 'Miguel Servet', Zaragoza (Spain), M Maghnie, IRCCS Policlinico S Matteo, University of Pavia (Italy), C Olivier (Serono), M Phillip, Schneider's Children Medical Center of Israel (Israel), M Ranke, Universitats-Kinderklinik, Tubingen (Germany), E Reiter, Tufts University School of Medicine, Springfield, IL (USA), R Ross, University of Sheffield (UK), M Savage, St Bartholomew's Hospital, London (UK), N Shaw, Birmingham Children's Hospital (UK), S M Webb, Autonomous University of Barcelona, Hospital Sant Pau, Barcelona (Spain), J M Wit, University Hospital,
Leiden (The Netherlands), H Wollmann (Pfizer) and H-J Zeisel (Sandoz).

\section{References}

1 Consensus guidelines for the diagnosis and treatment of adults with growth hormone deficiency, Summary statement of the Growth Hormone Research Society Workshop on Adult Growth Hormone Deficiency. Journal of Clinical Endocrinology and Metabolism $1998 \mathbf{8 3} 379-381$.

2 Growth Hormone Research Society, Consensus guidelines for the diagnosis and treatment of growth hormone $(\mathrm{GH})$ deficiency in childhood and adolescence: summary statement of the $\mathrm{GH}$ Research Society. Journal of Clinical Endocrinology and Metabolism $2000853990-3993$.

3 Tauber M, Jouret B, Cartault A, Lounis N, Gayrard M, Marcouyeux C, Pienkowski C, Oliver I, Moulin P, Otal P, Joffre F, Arnaud C \& Rochiccioli P. Adolescents with partial growth hormone $(\mathrm{GH})$ deficiency develop alterations of body composition after GH discontinuation and require follow-up. Journal of Clinical Endocrinology and Metabolism $2003 \mathbf{8 8} 5101-5106$.

4 Johannsson G, Albertsson-Wikland K \& Bengtsson BA. Discontinuation of growth hormone $(\mathrm{GH})$ treatment: metabolic effects in GH-deficient and GH-sufficient adolescent patients compared with control subjects. Swedish Study Group for Growth Hormone Treatment in Children. Journal of Clinical Endocrinology and Metabolism 199984 4516-4524.

5 Vahl N, Juul A, Jorgensen JO, Orskov H, Skakkebaek NE \& Christiansen JS. Continuation of growth hormone $(\mathrm{GH})$ replacement in GH-deficient patients during transition from childhood to adulthood: a two-year placebo-controlled study. Journal of Clinical Endocrinology and Metabolism 200085 1874-1881.

6 Colao A, Di Somma C, Salerno M, Spinelli L, Orio F \& Lombardi G. The cardiovascular risk of GH-deficient adolescents. Journal of Clinical Endocrinology and Metabolism 200287 3650-3655.

7 Drake WM, Carroll PV, Maher KT, Metcalfe KA, CamachoHubner C, Shaw NJ, Dunger DB, Cheetham TD, Savage MO \& Monson JP. The effect of cessation of growth hormone $(\mathrm{GH})$ therapy on bone mineral accretion in GH-deficient adolescents at the completion of linear growth. Journal of Clinical Endocrinology and Metabolism $2003 \mathbf{8 8} 1658-1663$.

8 Shalet SM, Shavrikova E, Cromer M, Child CJ, Keller E, Zapletalova J, Moshang T, Blum WF, Chipman JJ, Quigley CA \& Attanasio AF. Effect of growth hormone $(\mathrm{GH})$ treatment on bone in postpubertal GH-deficient patients: a 2 year randomized, controlled, dose-ranging study. Journal of Clinical Endocrinology and Metabolism 2003 88 4124-4129.

9 Underwood LE, Attie KM Baptista J \& Genentech Collaborative Study Group, Growth hormone $(\mathrm{GH})$ dose-response in young adults with childhood-onset GH deficiency: a two-year, multicenter, multiple-dose, placebo-controlled study. Journal of Clinical Endocrinology and Metabolism $2003 \mathbf{8 8} 5273-5280$.

10 Kelly JJ, Rajkovic IA, O’Sullivan AJ, Sernia C \& Ho KK. Effects of different oral oestrogen formulations on insulin-like growth factor-I, growth hormone and growth hormone binding protein in post-menopausal women. Clinical Endocrinology 199339 $561-567$.

11 Wolthers T, Hoffman DM, Nugent AG, Duncan MW, Umpleby M \& Ho KK. Oral estrogen antagonizes the metabolic actions of growth hormone in growth hormone-deficient women. American Journal of Physiology. Endocrinology and Metabolism $2001 \quad \mathbf{2 8 1}$ E1191-E1196.

12 GH Research Society, Critical evaluation of the safety of recombinant human growth hormone administration statement from the Growth Hormone Research Society. Journal of Clinical Endocrinology and Metabolism 200186 1868-1870.

13 Attanasio AF, Howell S, Bates PC, Frewer P, Chipman J, Blum WF \& Shalet SM. Body composition, IGF-I and IGFBP-3 concentrations as outcome measures in severely GH-deficient (GHD) 
patients after childhood GH treatment: a comparison with adult onset GHD patients. Journal of Clinical Endocrinology and Metabolism 200287 3368-3372.

14 Baroncelli GI, Bertelloni S, Sodini F \& Saggese G. Longitudinal changes of lumbar bone mineral density (BMD) in patients with $\mathrm{GH}$ deficiency after discontinuation of treatment at final height; timing and peak values for lumbar BMD. Clinical Endocrinology $200460175-184$.

15 Howlett TA. An assessment of optimal hydrocortisone replacement therapy. Clinical Endocrinology $1997 \mathbf{4 6} 263-268$.

16 Gelding SV, Taylor NF, Wood PJ, Noonan K, Weaver JU, Wood DF \& Monson JP. The effect of growth hormone replacement therapy on cortisol-cortisone interconversion in hypopituitary adults: evidence for growth hormone modulation of extrarenal 11 beta hydroxysteroid dehydrogenase activity. Clinical Endocrinology 199848 153-162.

17 Elsheikh M, Dunger DB, Conway GS \& Wass JA. Turner's syndrome in adulthood. Endocrine Reviews 200223 120-140.

18 Hogler W, Briody J, Moore B, Garnett S, Lu PW \& Cowell CT. Importance of estrogen on bone health in Turner syndrome: a cross-sectional and longitudinal study using dual-energy X-ray absorptiometry. Journal of Clinical Endocrinology and Metabolism 200489 193-199.

19 Van Pareren Y, Mulder P, Houdijk M, Jansen M, Reeser M \& Hokken-Koelega A. Adult height after long-term, continuous growth hormone $(\mathrm{GH})$ treatment in short children born small for gestational age: results of a randomized, double-blind, doseresponse GH trial. Journal of Clinical Endocrinology and Metabolism 200388 3584-3590.

20 Van Pareren YK, de Muinck Keizer-Schrama SM, Stijnen T, Sas TC, Jansen M, Otten BJ, Hoorweg-Nijman JJ, Vulsma T, StokvisBrantsma WH, Rouwe CW, Reeser HM, Gerver WJ, Gosen JJ, Rongen-Westerlaken C \& Drop SL. Final height in girls with Turner syndrome after long-term growth hormone treatment in three dosages and low dose estrogens. Journal of Clinical Endocrinology and Metabolism $2003 \mathbf{8 8} 1119-1125$.

Received 8 October 2004

Accepted 11 October 2004 